\title{
Third - Order Nonlinear Optical Properties and Optical Limiting Behavior of Celestin Blue B Dye Doped Polymer Films
}

\author{
Imad Al-Deen Hussein Ali Al-Saidi*, Raghad Jabar \\ Department of Physics, College of Education for Pure Sciences, University of Basrah, Basrah, Iraq \\ Email address: \\ al_saidi_imad@yahoo.com (I. Al-Deen H. A. Al-Saidi) \\ ${ }^{*}$ Corresponding author
}

To cite this article:

Imad Al-Deen Hussein Ali Al-Saidi, Raghad Jabar. Third - Order Nonlinear Optical Properties and Optical Limiting Behavior of Celestin Blue B Dye Doped Polymer Films. Journal of Photonic Materials and Technology. Vol. 4, No. 1, 2018, pp. 1-7.

doi: 10.11648/j.jmpt.20180401.11

Received: January 1, 2018; Accepted: January 30, 2018; Published: February 23, 2018

\begin{abstract}
Pure Poly (methylmethacrylate) (PMMA) polymer film and Celestin Blue B dye doped polymer films at different concentrations were prepared using casting technique. UV - Vis spectra were recorded to characterize the optical properties of the Celestin Blue B dye doped PMMA polymer films. The magnitudes of both real and imaginary parts of third - order nonlinear susceptibility $\left(\chi^{(3)}\right)$ of Celestin Blue B dye doped polymer films were determined using the $\mathrm{z}$ - scan technique with a continuous wave laser beam at wavelength $532 \mathrm{~nm}$. The nonlinear refractive index $\left(\mathrm{n}_{2}\right)$ and the nonlinear absorption coefficient $(\beta)$ of the Celestin Blue B dye doped polymer films were calculated. It was found that the prepared films exhibited saturable absorption (SA) and self - defocusing effect. The optical power limiting behavior also investigated for Celestin Blue B dye doped PMMA polymer films at different concentrations and the optical power limiting threshold values were determined. Results indicate that the Celestin Blue B dye doped PMMA polymer film is a promising material for future photonic device applications.
\end{abstract}

Keywords: Third - Order Nonlinearities, Nonlinear Optical Properties, Optical Power Limiting, Celestin Blue B Dye, Dye Doped Polymer Films, Z - Scan Technique

\section{Introduction}

Materials with high nonlinear optical (NLO) properties have received much attention recently due to their great potential in many important applications such as, optoelectronic and photonic devices, optical communications, optical signal processing, optical solar cells, optical sensors, light-emitting diodes (LED's), optical switching, and optical power limiting [1-15]. The protection of the human eyes and sensitive optical equipment from potentially intense laser beams are particularly important and required efficient control of the intensity of the incident laser beam. It has been found that the intensity of the laser beam can be controlled by using the optical power limiting properties of some nonlinear materials. Several materials have been investigated for their nonlinear optical (NLO) properties, and organic materials such as dyes and polymers have emerged as promising NLO materials [16 - 24]. These kinds of materials are found to exhibit good nonlinear optical properties and fast responses in the ultraviolet and visible (UV-Vis.) spectral domain. The wide range of polymer applications can be even more extended by the modification of their physical properties by the addition of organic dye to the polymer (as doping agent). Poly (methylmethacrylate) (PMMA) polymer, which we have chosen for the present work, is one of the important polymeric materials and it is widely used in various applications for its many advantageous properties such as: Highly light transmission (transparent material), flexible structure, ease of fabrication, good photo-thermal stability, and high damage threshold for the intense laser beams [10, $25,26]$, as well as it has a relatively low cost. PMMA polymer can be modified by incorporating different additives. These polymer modifications are typically 
performed to improve the optical and electrical properties of the polymer, targeted toward the photonic device applications. In the present work, Celestin Blue B dye has been used as an additive (or a dopant) material to PMMA polymer to obtain a structure in the form of dye doped polymer film. The results showed that the optical and electrical properties of the pure PMMA polymer are significantly affected by introducing the Celestin Blue B organic dye into PMMA polymer. Among the various techniques used to measure the optical parameters of nonlinear materials [27-32], the $\mathrm{z}$-scan technique [33, 34] proved to be an effective tool. This technique can be used to measure both nonlinear refractive index $\left(\mathrm{n}_{2}\right)$ and nonlinear absorption coefficient $(\beta)$ in materials. The obtained values of these parameters can be used to determine the magnitudes of both real and imaginary parts of third-order nonlinear susceptibility $\left(\chi^{(3)}\right)$ of Celestin Blue B dye doped PMMA polymer films. The z-scan technique can also be used to measure the optical power limiting behavior of Celestin Blue B dye doped PMMA polymer films.

In the current work, the nonlinear optical properties of Celestin Blue B dye doped PMMA polymer films at different concentrations were investigated using the $\mathrm{z}-\mathrm{scan}$ technique with $\mathrm{CW}$ laser beam. The nonlinear optical parameters such as, the nonlinear refractive index $\left(\mathrm{n}_{2}\right)$, the nonlinear absorption coefficient $(\beta)$, and the real and imaginary parts of third-order nonlinear susceptibility $\left(\chi^{(3)}\right)$ of Celestin Blue B dye doped PMMA polymer films were determined. Effect of variation the dye concentration on the values of these parameters was studied. The optical power limiting behavior of Celestin Blue B dye doped PMMA polymer films was also studied.

\section{Materials and Experimental Procedures}

\subsection{Preparation of the Samples}

The chemical structure of Celestin Blue $\mathrm{B}$ dye is shown in Figure 1 and its molecular formula is $\mathrm{C}_{17} \mathrm{H}_{18} \mathrm{Cl} \mathrm{N}_{3} \mathrm{O}_{4}$ with a molecular weight $\mathrm{M}_{\mathrm{W}}=363.80 \mathrm{~g} /$ mole. Poly (methylmethacrylate) (PMMA) polymer in the form of small grains (crystalline polymer) was used in this study. It has high purity and good optical transparency, with a molecular weight $\mathrm{M}_{\mathrm{w}}=84000 \mathrm{~g} /$ mole. Samples of dye - doped polymer films were prepared using the casting technique. Certain weight of Celestin Blue B dye powder was dissolved in a mixed solution of Tetrahydrofuran (THF) with a small quantity of methanol; this is suitable solvent for both dye and PMMA polymer. Then required weight of pure PMMA polymer was added. The mixture was stirred using a magnetic stirrer till a clear solution was formed. This solution was diluted by the solvent and samples of solutions with different dye concentrations were obtained. Proper quantities of the prepared solutions were poured on thin glass slides and kept for drying for 48 hours at room temperature. Dye doped polymer film samples of different concentrations $(0.05,0.06,0.07,0.08$, and $0.09 \mathrm{mM})$ and an average thickness of $0.9 \mathrm{~mm}$; were obtained. The prepared polymer films were examined carefully and found that are uniform with good optical quality.

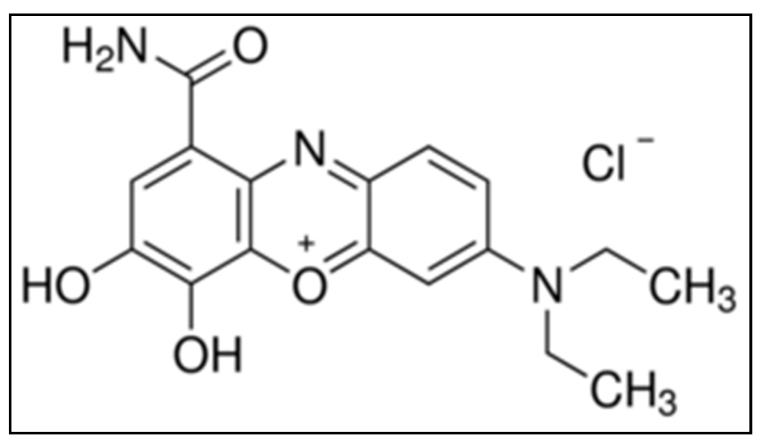

Figure 1. Chemical structure of Celestin Blue B dye.

\subsection{Experiments}

The $\mathrm{z}$ - scan experiments setup is shown in Figure 2. A linearly polarized Gaussian $\mathrm{TEM}_{00}$ beam of a continuous wave $(\mathrm{CW})$ solid - state laser (SSL) operating at wavelength $\lambda=532 \mathrm{~nm}$ was used. The power of the laser can be varied over the range $0-100 \mathrm{~mW}$. To perform the $\mathrm{z}$ - scan measurements, the laser Gaussian beam was focused into the sample to a beam waist $\omega_{0}=18 \mu \mathrm{m}$ using convex lens of focal length $5 \mathrm{~cm}$, producing a peak value of the on $\mathrm{z}$ - axis intensity at focus $\mathrm{I}_{0}=4.32 \mathrm{~kW} / \mathrm{cm}^{2}$. The Rayleigh range $\left(Z_{R}\right)$ (or the diffraction length) was calculated to be $1.91 \mathrm{~mm}$, and hence the sample of path $0.9 \mathrm{~mm}$ satisfies the thin medium condition in the $\mathrm{z}$ - scan technique, that is the sample thickness ( $\mathrm{L}$ ) less than the Rayleigh range (i.e., $\mathrm{n}_{0} \mathrm{~L}<\mathrm{Z}_{\mathrm{R}}$, where $n_{0}$ is the linear refractive index of the sample) [35]. An aperture of variable diameter placed in the far field is used to control the cross-section of the laser beam coming out of the sample. In our present work, we adjusted the diameter of the aperture as to get the optimum value of the linear transmittance, $\mathrm{S}=0.32$ (i.e., $32 \%$ of the laser beam transmission allowed to pass through the aperture). The sample was scanned across the focal plane of the lens along the propagation of the laser beam (the $\mathrm{z}$ - axis direction) using translation stage. The power of the transmitted laser beam through the aperture was measured by the photo detector $\mathrm{PD}_{2}$, coupled with digital power meter. The input power of the laser beam was measured by the photo-detector $\mathrm{PD}_{1}$, coupled with another digital power meter. The beam splitter (BS) was used to reflect part of the incident laser beam on the photo - detector $\mathrm{PD}_{1}$. 


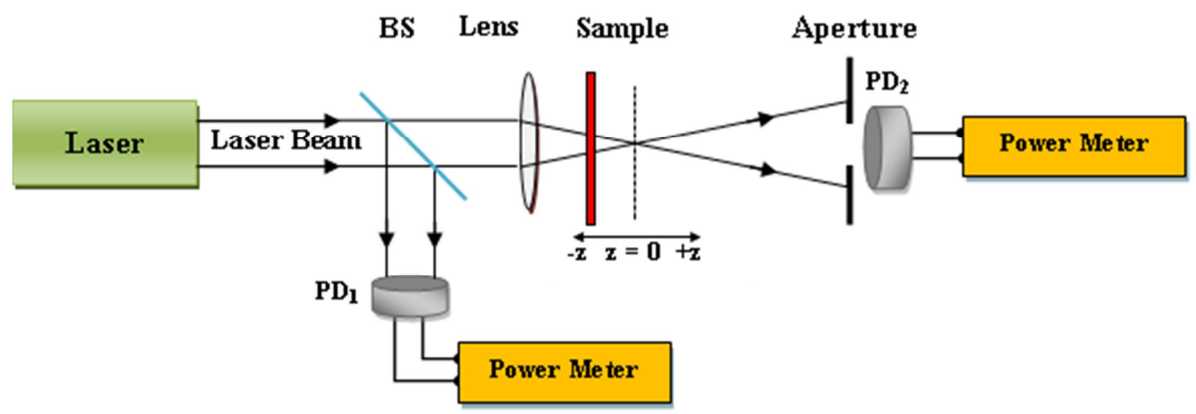

Figure 2. Experimental set-up for the $z$ - scan technique. BS, is a beam splitter; $P D_{1}$ and $P D_{2}$ are Photo - detectors.

\section{Results and Discussion}

\subsection{UV - Vis Spectroscopic Characterization}

The Ultraviolet - Visible (UV - Vis) absorbance spectra of the Celestin Blue B dye - doped polymer film samples at five different concentrations $(0.05,0.06,0.07,0.08$, and 0.09 $\mathrm{mM}$ ) were recorded over the wavelength range $300-900 \mathrm{~nm}$ using double - beam spectrophotometer (Cecil Model CE 7500 ) of the wavelength range $190-1100 \mathrm{~nm}$.

These spectra are shown in Figure 3. It is noticed that all the peaks of the absorbance curves are located at the wavelength $605 \mathrm{~nm}$ and the highest value of the measured optical absorbance is $16.8 \%$ for the dye - doped polymer sample with the dye concentration $0.09 \mathrm{mM}$. It is also noticed that the film samples exhibit high transmittance at low dye concentrations, and it is decreased as the dye concentration increases.

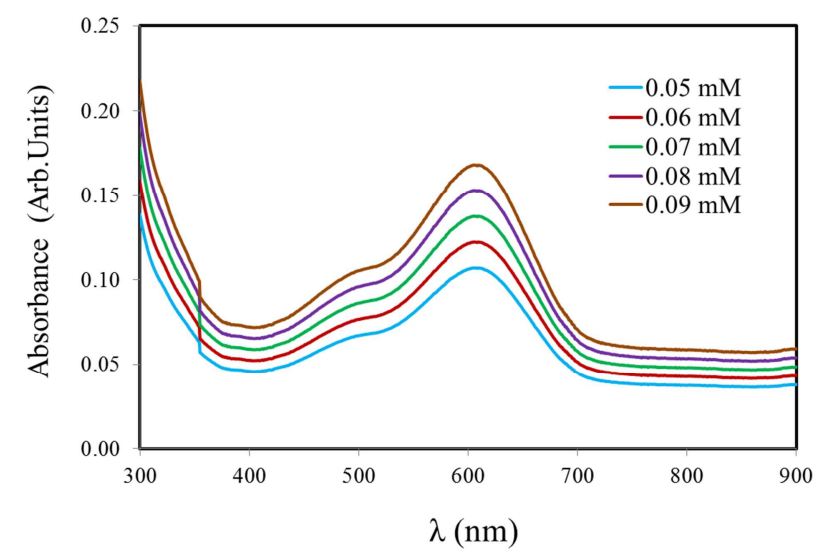

Figure 3. UV - Vis absorbance spectra of the Celestin Blue B dye-doped polymer films at different dye concentrations.

\subsection{Nonlinear Measurements}

The z - scan measurements for the Celestin Blue B dye doped polymer film at different dye concentrations are shown in Figure $4(a-c)$. Plot (a) is the closed - aperture $\mathrm{z}$ - scan and plot (b) is the open - aperture $\mathrm{z}$ - scan, where the normalized transmittance is plotted as a function of the sample position along the $\mathrm{z}$ - axis. It is clearly seen that the transmittance curves obtained are symmetric about focus for all sample concentrations, and this indicates that the studied sample follows the thermal nonlinear process. Since the closed- aperture transmittance is affected by both the nonlinear refraction and absorption, therefore it is necessary to separate the effect of the nonlinear refraction from the nonlinear absorption in order to determine the nonlinear refractive index $\left(\mathrm{n}_{2}\right)$. To obtain purely nonlinear refraction, the closed-aperture transmittance data are divided by the corresponding openaperture transmittance data [34]. The obtained pure nonlinear refraction curves for different dye concentrations are shown in Figure 4 (c). It is obvious from the closed-aperture, the film samples exhibited a transmission peak to valley is indicative of a negative nonlinear refractive index $\left(\mathrm{n}_{2}<0\right)$ due to selfdefocusing effect. This behavior is attributed to a thermal nonlinearity resulting from the local variation of refractive index (n) of the medium with temperature (T) (i. e., dn / dT). As a result, the medium acts as a thermal lens.
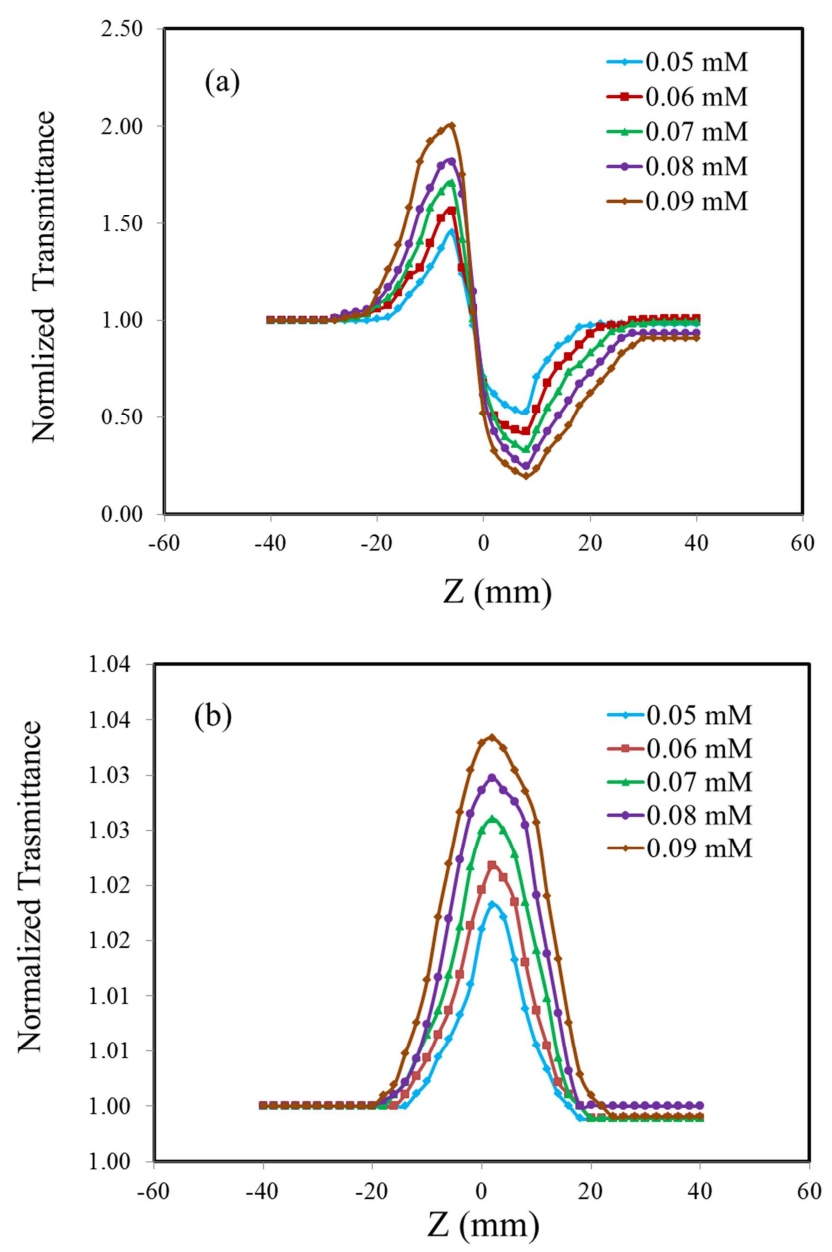


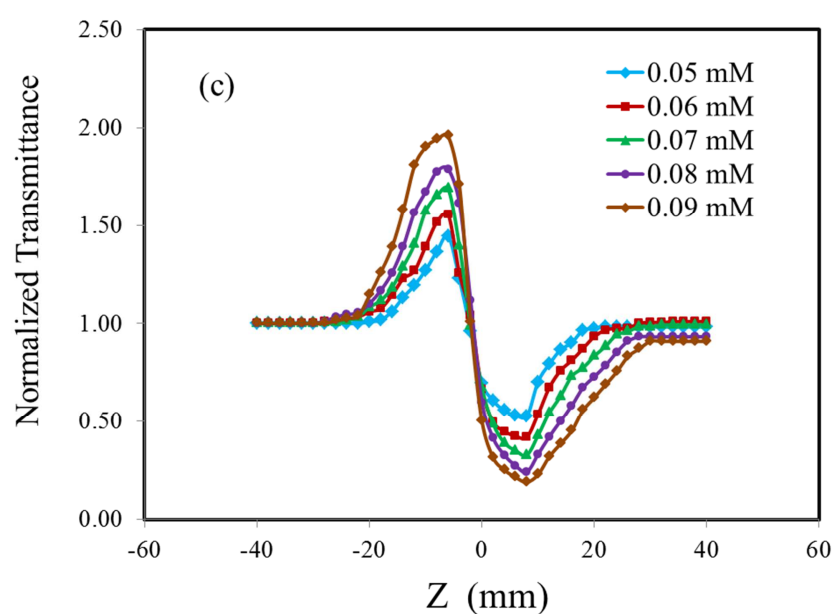

Figure 4. Normalized transmittance curves for the Celestin Blue B dyedoped polymer film at different dye concentrations. (a) Closed - aperture $z$ scan. (b) Open - aperture z-scan. (c) Pure nonlinear refraction.

Also, it is seen in the Figure 4 (a) that as the film sample is moved towards the focus (from $-\mathrm{z}$ to $+\mathrm{z}$ ), the intensity (transmittance) increases until reaches its maximum value at the focus $(\mathrm{z}=0)$, (valley position) indicating saturable absorption (SA) [36]. This suggests that the value of the nonlinear absorption coefficient $(\beta)$ is negative $(\beta<0)$.

The real part $\left(\operatorname{Re}\left(\chi^{(3)}\right)\right)$ and the imaginary part $\left(\operatorname{Im}\left(\chi^{(3)}\right)\right)$ of the third - order nonlinear optical susceptibility $\left(\chi^{(3)}\right)$ for the Celestin Blue B dye - doped polymer film were calculated from the estimated values of the nonlinear refractive index $\mathrm{n}_{2}$ (obtained from the closed - aperture $\mathrm{z}$ scan data) and the nonlinear absorption coefficient $\beta$ (obtained from the open - aperture $\mathrm{z}$ - scan data), using the following relations, respectively [37]:

$$
\begin{aligned}
& \operatorname{Re}\left[\chi^{(3)}\right](\mathrm{esu})=10^{-4} \frac{\varepsilon_{0} \mathrm{c}^{2} \mathrm{n}_{0}^{2}}{\pi} \mathrm{n}_{2}\left(\mathrm{~cm}^{2} / \mathrm{W}\right) \\
& \operatorname{Im}\left[\chi^{(3)}\right](\mathrm{esu})=10^{-2} \frac{\varepsilon_{0} \mathrm{c}^{2} \mathrm{n}_{0}^{2} \lambda}{4 \pi^{2}} \beta(\mathrm{cm} / \mathrm{W})
\end{aligned}
$$

where $\varepsilon_{0}$ is the permittivity of free space, $\mathrm{c}$ is the velocity of light in vacuum, and $\mathrm{n}_{0}$ is the linear refractive index of the medium.

The nonlinear refractive index $\left(\mathrm{n}_{2}\right)$ of the medium is given by the relation [35]:

$$
\mathrm{n}_{2}=\frac{\Delta \emptyset_{0} \lambda}{2 \pi \mathrm{I}_{0} \mathrm{~L}_{\mathrm{eff}}}
$$

and the change in the refractive index $(\Delta \mathrm{n})$ of the medium is given by the following relation $[35,38]$ :

$$
\Delta \mathrm{n}=\mathrm{n}_{2} \mathrm{I}_{0}
$$

where $\lambda$ is the laser wavelength and $I_{0}$ is the intensity of the laser beam at focus $(\mathrm{z}=0)$, and given by:

$$
\mathrm{I}_{0}=\frac{2 \mathrm{P}_{0}}{\pi \omega_{0}^{2}}
$$

where $\mathrm{P}_{0}$ is the laser input power. $\mathrm{L}_{\text {eff }}$ is the effective length of the sample and given by:

$$
\mathrm{L}_{\text {eff }}=\left(1-\exp \left(-\alpha_{0} \mathrm{~L}\right)\right) / \alpha_{0}
$$

where $\mathrm{L}$ is the true sample length and $\alpha_{0}$ is the linear absorption coefficient. $\Delta \varnothing_{0}$ in Eq. (3) is the on - axis phase shift of the laser beam traversing the medium. This phase shift is related to the difference between the peak and valley transmittances $\left(T_{p}\right.$ and $\left.T_{v}\right)$ for the closed - aperture $\mathrm{z}$ - scan $\Delta \mathrm{T}_{\mathrm{P}-\mathrm{v}}=\mathrm{T}_{\mathrm{p}}-\mathrm{T}_{\mathrm{v}}$ according to the following relation $[33,34]$ :

$$
\Delta \mathrm{T}_{\mathrm{P}-\mathrm{v}}=0.406(1-\mathrm{S})^{0.25}\left|\Delta \emptyset_{0}\right|
$$

where $\left|\Delta \varnothing_{0}\right|$ is the on - axis nonlinear phase shift at the focus and $\mathrm{S}$ is the linear transmittance of the aperture and given by:

$$
\mathrm{S}=1-\exp \left(-2 \mathrm{r}_{\mathrm{a}}^{2} / \omega_{\mathrm{a}}^{2}\right)
$$

where $r_{a}$ is the radius of the aperture and $\omega_{a}$ is the radius of the laser beam at the entrance of the aperture.

The nonlinear absorption coefficient $(\beta)$ can be calculated from the open - aperture $\mathrm{z}$ - scan data and using the following relation:

$$
\beta=\frac{2 \sqrt{2}}{I_{0} L_{e f f}} \Delta T
$$

where $\Delta \mathrm{T}$ is the normalized transmittance difference between peak at the focal point $(\mathrm{z}=0)$ in the open - aperture $\mathrm{z}$ - scan normalized transmittance curve and the baseline.

The absolute value of the third - order nonlinear optical susceptibility was calculated from the following relation:

$$
\left|\chi^{(3)}\right|=\left[\left(\operatorname{Re}\left(\chi^{(3)}\right)\right)^{2}+\left(\operatorname{Im}\left(\chi^{(3)}\right)\right)^{2}\right]^{1 / 2}
$$

Figures 5, 6, 7, 8, and 9, respectively, illustrate the variations of $\Delta \mathrm{T}_{\mathrm{P}-\mathrm{v}}, \mathrm{n}_{2}, \Delta \mathrm{n}, \beta$, and $\left|\chi^{(3)}\right|$ as a function of the Celestin Blue $\mathrm{B}$ dye concentration. It is clearly seen that the values of $n_{2}$ and $\beta$ increase as the dye concentration increases. This may be attributed to the fact that, as the dye concentration increases the number of dye molecules increases and more particles are thermally gaited resulting in an enhanced effect. The calculated values of the $\Delta \mathrm{T}_{\mathrm{P}-\mathrm{v}}, \mathrm{n}_{2}$, $\Delta \mathrm{n}, \beta$, and $\left|\chi^{(3)}\right|$ are listed in Table (1).

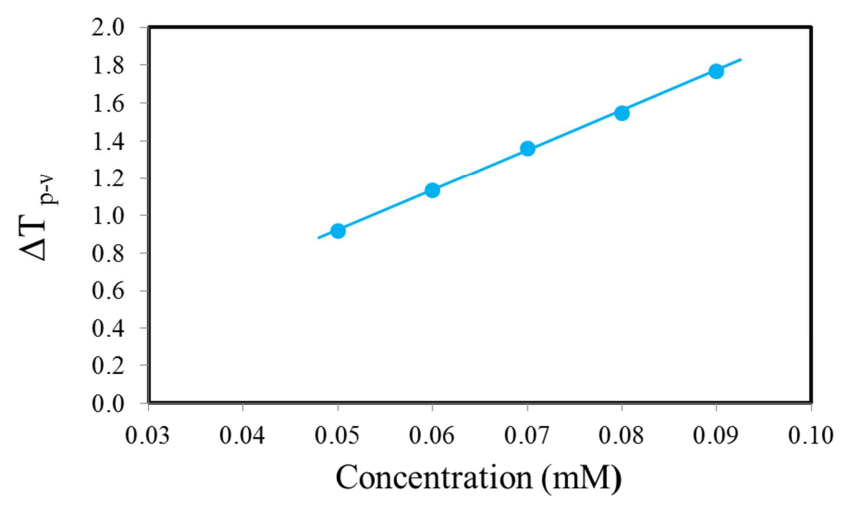

Figure 5. Variation of $\Delta T_{P-v}$ as a function of the Celestin Blue $B$ dye concentration. 


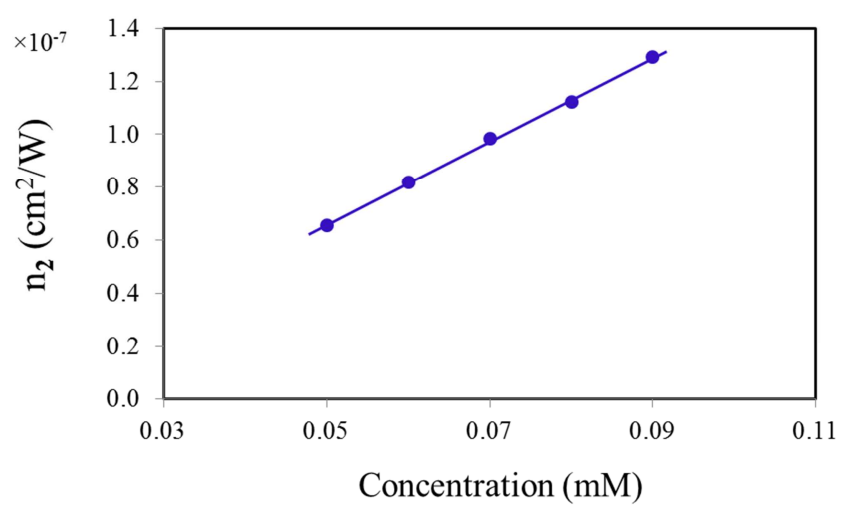

Figure 6. Variation of $n_{2}$ as a function of the Celestin Blue $B$ dye concentration.

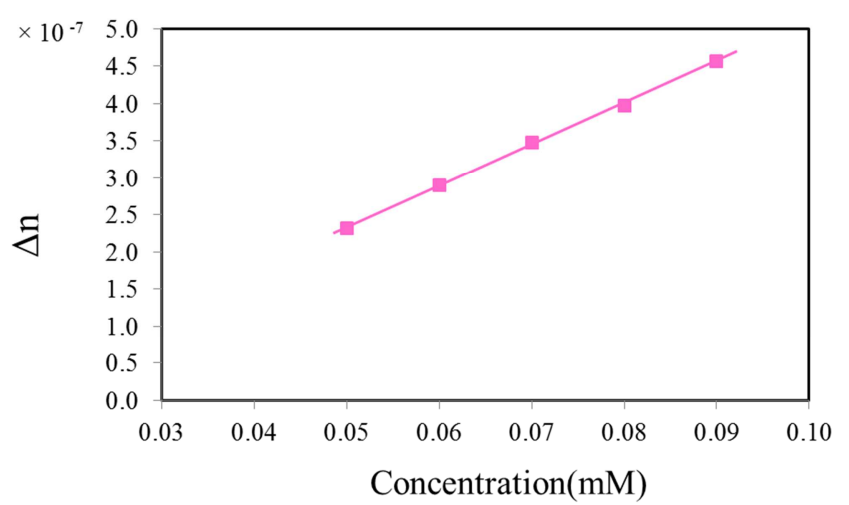

Figure 7. Variation of $\Delta n$ as a function of the Celestin Blue $B$ dye concentration.

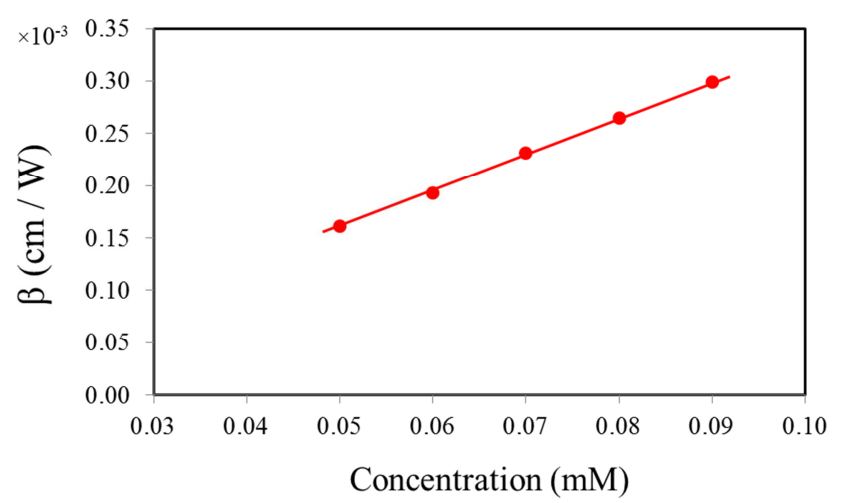

Figure 8. Variation of $\beta$ as a function of the Celestin Blue $B$ dye concentration.

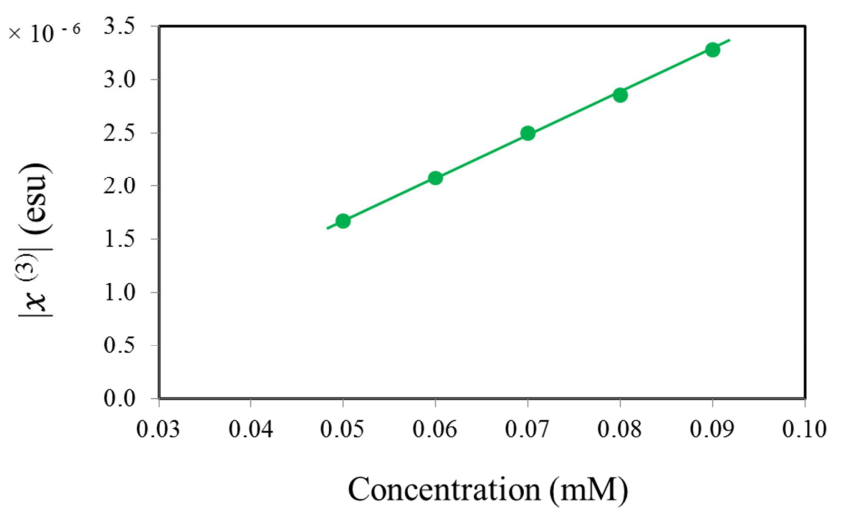

Figure 9. Variation of $\left|\chi^{(3)}\right|$ with the Celestin Blue B dye concentration.

Table 1. The calculated values of the nonlinear optical parameters of Celestin Blue B dye - doped polymer film for five different dye concentrations.

\begin{tabular}{llllll}
\hline Concentration $(\mathbf{m M})$ & $\mathbf{\Delta} \mathbf{T}_{\mathbf{P}-\mathbf{V}}$ & $\mathbf{n}_{\mathbf{2}}\left(\mathbf{c m}^{\mathbf{2}} / \mathbf{W}\right) \times \mathbf{1 0}^{-\mathbf{7}}$ & $\mathbf{\Delta} \mathbf{n} \times \mathbf{1 0}^{-4}$ & $\boldsymbol{\beta}(\mathbf{c m} / \mathbf{W}) \times \mathbf{1 0}^{-\mathbf{3}}$ & $\left|\chi^{(3)}\right|(\mathbf{e s u}) \times \mathbf{1 0}^{-\mathbf{6}}$ \\
\hline 0.05 & 0.91 & -0.65 & -2.32 & -0.16 & 1.66 \\
0.06 & 1.13 & -0.81 & -2.89 & -0.19 & 2.07 \\
0.07 & 1.36 & -0.98 & -3.47 & -0.23 & 2.49 \\
0.08 & 1.54 & -1.12 & -3.97 & -0.26 & 2.85 \\
\hline
\end{tabular}

Optical power limiting behavior of Celestin Blue B dye doped polymer film at different dye concentrations was studied. The laser output power is plotted as a function of the laser input power for different dye concentrations, as shown in Figure 10. It is seen that the laser output power rises initially with increase in laser input power, but after a certain laser input power value, which is called the optical power limiting threshold $\left(\mathrm{P}_{\mathrm{th}}\right)[39,40]$, the medium (the sample) starts to exhibits self - defocusing effect, resulting in a larger part of laser beam cut off by the aperture. Consequently the transmitted beam recorded by the photo - detector $\mathrm{D}_{2}$ remained approximately constant. The optical power limiting behavior is significantly affected by the variation of the dye concentration, the sample with low dye concentration shows a weak optical power limiting while the sample with high dye concentration exhibits strong optical power limiting.

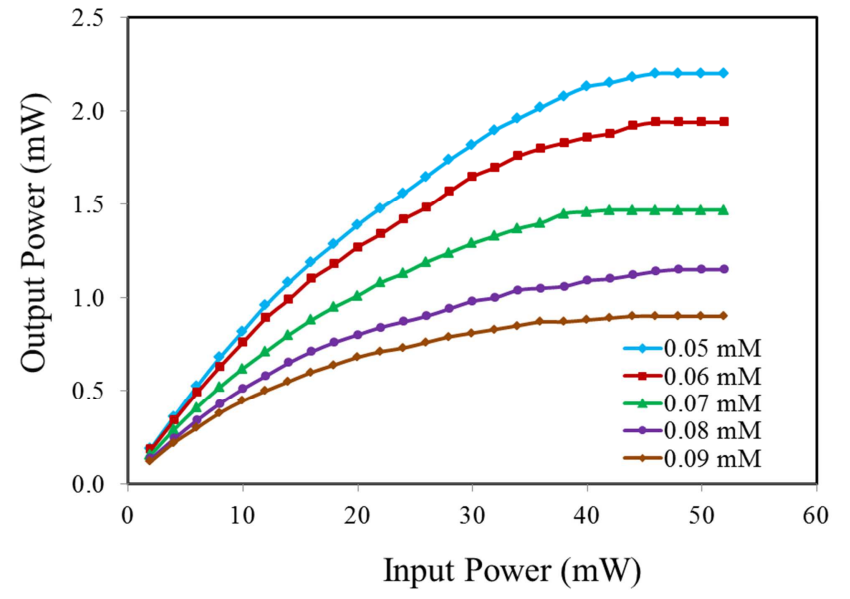

Figure 10. Optical power limiting behavior (laser output power as a function of laser input power) for Celestin Blue B dye - doped polymer film at different concentrations.

Values of the optical power limiting threshold $\left(\mathrm{P}_{\text {th }}\right)$ were 
estimated from Figure 10, for the Celestin Blue B dye - doped polymer film. Figure 11 illustrates the relation between the optical power limiting threshold $\left(\mathrm{P}_{\text {th }}\right)$ and the dye concentration. It is clearly evident that the optical power limiting threshold linearly decreases with increasing the dye concentration. This is due to the fact that the number of absorbing molecules increases with increasing the dye concentration which in turn lowers the optical power limiting threshold. We can deduce from the observed results that the dye concentration plays an important role in controlling the power limiting behavior. Also, the incident intense laser beam can significantly change the absorptive and refractive properties of the medium (the sample) resulting in a greatly reduced output intensity and therefore it is important to determine the magnitude of the medium nonlinearity to find the suitable conditions for the optical power limiting behavior.

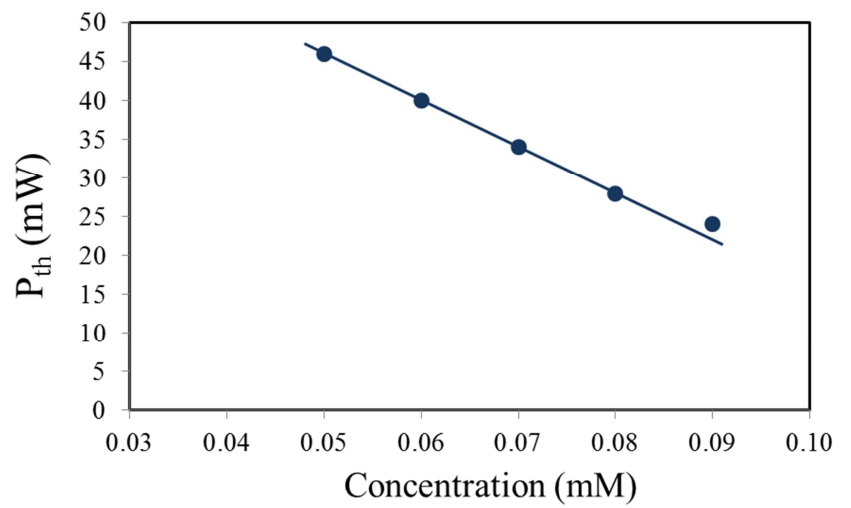

Figure 11. Optical Power limiting threshold $\left(P_{t h}\right)$ of Celestin Blue B dye doped polymer film as a function of dye concentration.

\section{Conclusions}

Pure poly (methylmethacrylate) (PMMA) polymer film and Celestin Blue B dye - doped polymer films at different concentrations were prepared using casting technique. The third - order nonlinear optical properties and optical power limiting behavior of Celestin Blue B dye - doped polymer films have been investigated. The optical parameters, $\Delta \mathrm{T}_{\mathrm{P}-\mathrm{v}}, \mathrm{n}_{2}$, $\Delta \mathrm{n}, \beta$, and $\left|\chi^{(3)}\right|$ were measured using the $\mathrm{z}$ - scan technique with a continuous wave laser beam at wavelength $532 \mathrm{~nm}$. The experimental results showed that Celestin Blue B dye - doped polymer film has a large third - order optical nonlinearities. The origin of the nonlinear effects is attributed to the thermal variation of refractive index of the medium. It is found that the optical power limiting depends on the dye concentration and possesses low optical power limiting threshold. From the results, the Celestin Blue B dye - doped polymer film is expected to be promising candidate for applications on nonlinear photonic devices and optical limiters.

\section{References}

[1] M. Bass, (Ed.), Handbook of Optics: Optical Properties of Materials, Nonlinear Optics, Quantum Optics, Vol. IV, Third Edition, (The McGraw - Hill Companies, Inc., New York, 2010).
[2] H. S. Nalwa, (Ed.), Handbook of Advanced Electronic and Photonic Materials and Devices, (Academic Press, New York, USA, 2001).

[3] S. O. Kasap and R. K. Sinha, Optoelectronics and Photonics: Principles and Practices, Vol. 340, (Prentice Hall, New Jersey, USA, 2001).

[4] Z. M. Wang and A. Neogi, (Eds.), Nanoscale Photonic and Optoelectronics, (Springer - Verlag, Heidelberg / Berlin, Germany, 2010).

[5] G. I. Stegeman and R. A. Stegeman, Nonlinear Optics: Phenomena, Materials, and Devices, (John Wiley and Sons, Inc., Publication, New Jersey, USA, 2012).

[6] O. Wada, "Femtosecond All - Optical Devices for Ultrafast Communication and Signal Processing", New J. Phys., 6 (2004) 183-185.

[7] J. M. Hales, S. Zheng, S. Barlow, S. R. Marder, and J. W. Perry, "Bisdioxaborine Polymethines with Large Third - Order Nonlinearities for All - Optical Signal Processing", J. Amer. Chem. Soc., 128 (2006)11362-11363.

[8] A. Sacco, M. Gerosa, S. Bianco, L. Mercatelli, R. Fontana, L. Pezzati, L. Quaglio, C. F. Dirri, and A. O. M. Tucci, "Dye Sensitized Solar Cell for a Solar Concentrator System, Solar Energy, 125 (2016) 307-313.

[9] G. Hasanyi, Polymer Films in Sensor Applications; Technology, Materials, Devices, and Their Applications, (Technomic Publishing Company, Inc., Pennsylvania, USA, 1995).

[10] S. -L. Yeh, C. -Y. Zhu, and S. -W. Kuo, Transparent Heat Resistant PMMA Copolymers for Pacing Light-Emitting Diode Materials, Polymers, 7 (2015) 1379-1388.

[11] F. Z. Henri and S. Cassidy, "Nonlinear Optical Properties and All - Switching of Gongo Red in Solution", Optik, 123 (2012) 711-714.

[12] Z. Chai, X. Hu, F. Wang, X. Niu, J. Xie, and Q. Gong, "Ultrafast All - Optical Switching", Adv. Optical Mater., 5 (2017) 665-685.

[13] I. Al-D. H. A. Al-Saidi and S. Al-D. Abdulkareem, "Nonlinear Optical Properties and Optical Power Limiting of Leishman Dye Using Z-Scan Technique", J. Mater Sci.: Mater Electron, 26 (2015) 2713-2718.

[14] I. Al-D. H. A. Al-Saidi and S. Al-D. Abdulkareem, "Nonlinear Optical Properties and Optical Power Limiting Behavior of Leishman Dye in Solution and Solid Polymer Film Using Z-Scan", Optik Inter. J. Light and Elec. Optics, 126 (2015) 4299-4303.

[15] I. Al-D. H. A. Al-Saidi and S. Al-D. Abdulkareem, "Nonlinear Optical Properties and Optical Power Limiting Effect of Giemsa Dye Polymer Films", Opt. Laser Technol., 82 (2016) 150-156.

[16] H. S. Nalwa and S. Miyata (Eds.), Nonlinear Optics of Organic Molecules and Polymers, (CRC Press, Inc., New York, USA, 1997).

[17] P. Günter, (Ed.), Nonlinear Optical Effects and Materials, (Springer - Verlag, Heidelberg / Berlin, Germany, 2000).

[18] H. Zollinger, Color Chemistry: Synthesis, Properties and Applications of Organic Dyes and Pigments, Third Edition, (Wiley - VCH GmbH and Co. KGaA, Weinheim, Germany, 2003). 
[19] K. Hunger (Ed.), Industrial Dyes: Chemistry, Properties, Applications, (Wiley - VCH, GmbH and Co. KGaA, Weinheim, Germany, 2003).

[20] J. E. Mark (Ed.), Physical Properties of Polymers Handbook, (Springer, Dordrecht, Netherlands, 2007).

[21] A. A. Ishchenko, "Photonics and Molecular Design of DyeDoped Polymers for Modern Light-Sensitive Materials", Pure Appl. Chem., 80 (2008) 1525-1538.

[22] R. A. Ganeev, Nonlinear Optical Properties of Materials, Springer Series in Optical Sciences, Vol. 174, (Springer, Dordrecht, Netherlands, 2013).

[23] O. Ostroverkhova, (Ed.), Handbook of Organic Materials for Optical and Opto- electronic Devices: Properties and Applications, (Woodhead Publishing Ltd., UK, 2013).

[24] S. - S. Sun and L. R. Dalto, (Eds.), Introduction to Organic Electronic and Optoelectronic: Materials and Devices, (CRC Press, Boca Raton, USA, 2017).

[25] R. Gupta, V. Kumar, P. K. Goyal, and S. Kumar,”Optical Characterization of Poly (methylmethacrylate) Implanted with Low Energy Ions”, Appl. Surf. Sci., 263 (2012) 334-338.

[26] A. Pal and P. K. Khare, "Elecrical Conductivity Behavior of Pure and Polyblends Samples of Polyvinyl Chloride (PVC) and Polymethyl methacrylate (PMMA)", J. Electrost., 71 (2013) 976-986.

[27] W. E. Williams, M. J. Soileau, and E. W. Van Stryland, Optical Switching and $\mathrm{n}_{2}$ Measurements in $\mathrm{CS}_{2}$, Opt. Commun., 50 (1984) 256-260.

[28] R. Adair, L. L. Chase, and S. A. Payne, "Nonlinear Refractive - Index Measurements of Glasses Using Three-Wave Frequency Mixing”, J. Opt. Soc. Am. B, 4 (1987) 875-881.

[29] G. Boudebs, M. Chis, and J. P. Bourdin, Third-Order Susceptibility Measurements by Nonlinear Image Processing, J. Opt. Soc. Am. B, 13 (1996) 1450-1456.

[30] P. Vasaa, B. P. Singh, P. Tanejaa, and P. Ayyub, “Antiresonant Ring Interferometry as a Sensitive Technique for Measuring Nonlinear Optical Properties of Thin Films", Opt. Commun., 233 (2004) 297-304.
[31] Z. - B. Liu, X. - Q. Yan, J. - G. Tian, W.-Y. Zhou, and W.- P. Zang, "Nonlinear Ellipse Rotation Modified Z - Scan Measurements of Third - Order Nonlinear Susceptibility Tensor", Opt. Express, 15 (2007) 13351-13359.

[32] R. S. S. Kumar, S. V. Rao, L. Giribabu, and D. N. Rao, "Ultrafast Nonlinear Optical Properties of Alkyl Phthalocyanines Investigated Using Degenerate Four - Wave Mixing Technique”, Opt. Mater., 31 (2009) 1042-1047.

[33] M. Sheik - Bahae, A. A. Said, and E. W. Van Stryland "High Sensitivity, Single- Beam $\mathrm{n}_{2}$ Measurements", Opt. Lett., 14 (1989) 955-957.

[34] M Sheik - Bahae, A A Said, T Wei, D J Hagan, and E W Van Stryland, "Sensitive Measurement of Optical Nonlinearities Using a Single Beam", IEEE J. Quantum Electron., QE - 26 (1990) 760-769.

[35] E. W. Van Stryland and M. Sheik - Bahae, "Z - Scan Measurements of Optical Nonlinearities", in Characterization Techniques and Tabulations for Organic Nonlinear Materials, M. G. Kuzyk and C. W. Dirk, (Eds.), (Marcel Dekker, Inc., New York, 1998) 655-692.

[36] R. L. Sutherland, (Ed.), Handbook of Nonlinear Optics, Second Edition, (Marcel Dekker, New York, USA, 2003).

[37] T Cassano, R Tommasi, M Ferrara, F Babudri, G M Farinola, and F. Naso, "Substituent - Dependence of the Optical Nonlinearities in Poly (2,5 - Diakoxy - P - Phenylenevinylene) Polymers Investigated by Z - Scan Technique, Chem. Phys., 272 (2001)111-118.

[38] R. W. Boyd, Nonlinear Optics, Third Edition, (Elsevier Inc., New York, USA, 2008).

[39] W. L. Tan, W. Ji, J. L. Zuo, J. F. Bai, X. Z. You, J. H. Lim, S. Yang, D. J. Hagan, and E. W. Van Stryland, "Optical-Limiting Properties of Neutral Nickel Dithiolenes," Appl. Phys. B, 70 (2000) 809-812.

[40] A. Nevejina - Sturhan, O. Werhahn, and U. Siegner, "Low Threshold High - Dynamic - Range Optical Limiter for Ultrashort Laser Pulses,” Appl. Phys. B, 74 (2002) 553-557. 